\title{
Shikonin reduces hepatic fibrosis by inducing apoptosis and inhibiting autophagy via the platelet-activating factor-mitogen-activated protein kinase axis
}

\author{
MIN SONG, HENG ZHANG, ZHITAO CHEN, JING YANG, JIE LI, SUE SHAO and JING LIU \\ Department of Gastroenterology, The Central Hospital of Wuhan, Tongji Medical College, \\ Huazhong University of Science and Technology, Wuhan, Hubei 430014, P.R. China
}

Received January 15, 2020; Accepted July 23, 2020

DOI: $10.3892 /$ etm.2020.9460

\begin{abstract}
Liver fibrosis is a tissue repair process that occurs following various types of chronic liver injury and can develop into liver cirrhosis, portal hypertension or liver cancer without effective treatment. Shikonin has anti-inflammatory, antiviral and antitumor properties. Furthermore, shikonin has an additional effect of antagonizing tissue and organ fibrosis. The aim of the present study was to evaluate the mechanisms of action underlying shikonin against liver fibrosis. Cell viability was assessed using the Cell Counting Kit- 8 and EdU incorporation assays. Protein and mRNA expression levels were measured via western blotting and immunofluorescence assays, respectively. Apoptosis was examined via flow cytometry and autophagy via transmission electron microscopy. Compared with the control group, shikonin did not significantly alter LX-2 cell viability at $0.2 \mu \mathrm{mol} / \mathrm{ml}$, which was used as the intervention concentration. However, shikonin significantly inhibited fibrosis, as indicated by a decrease in the expression of $\alpha$-smooth muscle actin and collagen-I in the TGF- $\beta+$ shikonin group compared with the TGF- $\beta$ group. The results indicated that shikonin potentially inhibited fibrosis via promoting cell apoptosis and inhibiting autophagy. Additionally, the results of the present study indicated that shikonin downregulated the expression levels of platelet-activating factor (PAF) in TGF- $\beta$-treated cells, which subsequently activated the MAPK signaling pathway, leading to enhanced cell apoptosis and reduced autophagy.
\end{abstract}

Correspondence to: Professor Jing Liu, Department of Gastroenterology, The Central Hospital of Wuhan, Tongji Medical College, Huazhong University of Science and Technology, 26 Shengli Street, Wuhan, Hubei 430014, P.R. China

E-mail:2838795935@qq.com

Abbreviations: PAF, platelet-activating factor; HSC, hepatic stellate cell; ECM, extracellular matrix

Key words: liver fibrosis, cell apoptosis, autophagy, platelet-activating factor-MAPK signaling pathway
Collectively, the present study indicated that shikonin promoted cell apoptosis and suppressed autophagy via the PAF-MAPK axis in LX-2 cells, thus blocking the development of fibrosis. The results of the present study may provide a potential therapeutic strategy for liver fibrosis.

\section{Introduction}

Liver fibrosis is a tissue repair response to various types of chronic liver injury and a common pathological process among all chronic liver diseases (1). Without effective treatment, liver fibrosis can develop into liver cirrhosis, portal hypertension and even liver cancer (1), which can seriously affect the quality of life of patients. Liver fibrosis is characterized by the excessive generation and deposition of extracellular matrix (ECM), including type I collagen (COL-I) and fibronectin, in the liver parenchyma, resulting in damage to liver structure and function (2,3). Basic and clinical studies have reported that activated hepatic stellate cells (HSCs), namely myofibroblasts, are the primary source of ECM in the process of liver fibrosis (2,3).

Shikonin is a naphthoquinone compound derived from the root of Lithospermum erythrorhizon (4). In addition to its previously reported anti-inflammatory, antiviral and antitumor properties, shikonin exerts an effect against tissue and organ fibrosis (5). For example, Nie et al (6) reported that shikonin inhibited lung fibroblast proliferation and activation by regulating the Akt/P38 signaling pathway. Ding et al (7) demonstrated that shikonin inhibited the activation of renal mesenchymal fibroblasts and renal fibrosis by inhibiting aerobic glycolysis. Additionally, Fan et al (8) observed that shikonin reduced TGF- $\beta 1$-induced collagen production and contraction in hypertrophic scars derived from human skin fibroblasts. Yang et al (9) reported that shikonin alleviated isoproterenol-induced myocardial injury by inhibiting fibrosis, apoptosis, inflammation and endoplasmic reticulum stress. However, the action and mechanisms underlying shikonin in the prevention of liver fibrosis are not completely understood. Therefore, the present study aimed to investigate the effects of shikonin on liver fibrosis and explore the possible underlying mechanisms. 


\section{Materials and methods}

Cell lines and cell culture. The classic HSC line LX-2 was obtained from the American Type Culture Collection. Cells were cultured in DMEM supplemented with $10 \%$ FBS, $100 \mathrm{mg} / \mathrm{ml}$ penicillin and $100 \mathrm{U} / \mathrm{ml}$ streptomycin (all from Gibco; Thermo Fisher Scientific, Inc.) at $37^{\circ} \mathrm{C}$ with $5 \% \mathrm{CO}_{2}$. To induce cell activation, $\mathrm{LX}-2$ cells $\left(1 \times 10^{5}\right.$ cells/well $)$ were incubated with TGF- $\beta$ ( $5 \mathrm{ng} / \mathrm{ml}$; PeproTech, Inc.) for $24 \mathrm{~h}$ at $37^{\circ} \mathrm{C}$.

Cell viability assay. $\mathrm{LX}-2$ cells $\left(1 \times 10^{3}\right.$ cells/well) were seeded into 96-well plates and treated with shikonin (10 $\mu \mathrm{mol}$; 99.8\%; MedChemExpress.) for $24 \mathrm{~h}$ at $37^{\circ} \mathrm{C}$. Subsequently, Cell Counting Kit-8 (CCK-8; BioSharp Life Sciences) reagent was added for $4 \mathrm{~h}$ at $37^{\circ} \mathrm{C}$, according to the manufacturer's protocol. The absorbance of each well was measured at a wavelength of $450 \mathrm{~nm}$ using a microplate reader (Thermo Fisher Scientific, Inc.). DNA synthesis was measured by performing an EdU incorporation assay (Shanghai Yeasen Biotechnology Co., Ltd.), according to the manufacturer's protocol. Cells were visualized using a fluorescence microscope (magnification, x200; Nikon Corporation).

Western blotting. LX-2 cells ( $1 \times 10^{5}$ cells/well) were seeded in 6 -well plates. After culturing overnight, cells were stimulated with TGF- $\beta(5 \mathrm{ng} / \mathrm{ml})$ for $24 \mathrm{~h}$ at $37^{\circ} \mathrm{C}$, followed by treatment with shikonin $(0.2 \mu \mathrm{mol} / 1)$ for $24 \mathrm{~h}$ at $37^{\circ} \mathrm{C}$. Total protein was extracted using RIPA cell lysis buffer (Sigma-Aldrich; Merck KGaA) containing a protease inhibitor mixture (Sigma-Aldrich; Merck KGaA) for $30 \mathrm{~min}$. Subsequently, total protein was quantified using the BCA method. Proteins (50 $\mu \mathrm{g} /$ lane) were separated via $12 \%$ SDS-PAGE and transferred onto PVDF membranes (Merck KGaA). After blocking with 5\% BSA (BD Biosciences) for $1 \mathrm{~h}$ at room temperature, the membranes were incubated overnight at $4^{\circ} \mathrm{C}$ with the following primary antibodies: anti- $\alpha$-smooth muscle actin $(1: 1,000$; cat. no. ab7817), anti-Bcl-2 (1:1,000; cat. no. ab59384), anti-Bax (1:1,000; cat. no. ab32503), anti-light chain 3 (LC3)-II/I (1:1,000; cat. no. ab192890), anti-cleaved-caspase-3 (1:1,000; cat. no. ab49822), anti-caspase-3 (1:1,000; cat. no. ab13847), anti-Beclin-1 (1:1,000; cat. no. ab210498), anti-COL-I (1:1,000; cat. no. ab260043), anti-P62 (1:1,000; cat. no. ab56416), anti-JNK (1:1,000; cat. no. ab76125), anti-phosphorylated (p)-JNK (1:1,000; cat. no. ab76572), anti-P38 (1:1,000; cat. no. ab31828), anti-p-P38 (1:1,000; cat. no. ab47363) and anti-platelet-activating factor (PAF; 1:1,000; cat. no. ab104162). Subsequently, the membranes were incubated with secondary antibodies for horseradish peroxidase-conjugated goat anti-rabbit immunoglobulin G $(1: 5,000$; cat. no. ab6721) or goat anti-mouse IgG (1:5,000; cat. no. ab6728) for $2 \mathrm{~h}$ at room temperature. All primary and secondary antibodies were purchased from Abcam. Protein bands were visualized using ECL chemiluminescence (Merck KGaA) and analyzed using Image-Pro Plus software (version 6.0; Media Cybernetics, Inc.). GAPDH (Abcam; 1:1,000; cat. no. ab9485) was used as the loading control.

Immunofluorescence. $\mathrm{LX}-2$ cells $\left(1 \times 10^{5}\right.$ cells/well) were seeded onto glass coverslips in 6-well plates. After culturing overnight, cells were stimulated with TGF- $\beta(5 \mathrm{ng} / \mathrm{ml})$ for $24 \mathrm{~h}$ at $37^{\circ} \mathrm{C}$, followed by treatment with shikonin $(0.2 \mu \mathrm{mol} / 1)$ for $24 \mathrm{~h}$ at $37^{\circ} \mathrm{C}$. Cells were fixed with $4 \%$ paraformaldehyde for $15 \mathrm{~min}$ at room temperature. Cells were rinsed twice with PBS, permeabilized with $0.1 \%$ Triton X-100 in PBS for $30 \mathrm{~min}$ at $4{ }^{\circ} \mathrm{C}$ and blocked with 5\% BSA in PBS for $1 \mathrm{~h}$ at room temperature. Cells were incubated with LC3-II/I primary antibodies (Abcam; 1:1,000; cat. no. ab192890) overnight at $4^{\circ} \mathrm{C}$. After rinsing three times with PBS, cells were incubated with an Alexa Fluor-568-conjugated secondary anti-rabbit IgG antibodies (1:250; Invitrogen; Thermo Fisher Scientific, Inc.) for $1 \mathrm{~h}$ at room temperature. Subsequently, cells were counterstained with DAPI (Beijing Solarbio Science \& Technology Co., Ltd.) for $10 \mathrm{~min}$ at room temperature to detect nuclei. Stained cells were observed using an LSM410 confocal laser scanning microscope (magnification, x200; Carl Zeiss AG) and analyzed using Image-Pro Plus software (version 6; Media Cybernetics, Inc.).

Flow cytometric analysis of cellular apoptosis. LX-2 cell apoptosis was determined via flow cytometry using an Annexin V-FITC/propidium iodide (PI) kit (BD Biosciences), according to the manufacturer's protocol. Briefly, LX-2 cells $\left(1 \times 10^{5}\right.$ cells/well) were collected and resuspended in $200 \mu 1$ binding buffer with Annexin V and PI. Following treatment with shikonin for $24 \mathrm{~h}$, cells were incubated at room temperature for $20 \mathrm{~min}$ and analyzed by flow cytometry using a FACSCalibur flow cytometry (BD Biosciences).

Transmission electron microscopy (TEM). LX-2 cells were maintained in $2.5 \%$ glutaraldehyde for $12 \mathrm{~h}$ at $4{ }^{\circ} \mathrm{C}$ and fixed with $1 \%$ osmic acid for $3 \mathrm{~h}$ at $4^{\circ} \mathrm{C}$. Cells were dehydrated and embedded in epoxy resin for $4 \mathrm{~h}$ at room temperature. Subsequently, cells were stained with uranyl acetate and lead citrate. Cells were observed using TEM (Leica Microsystems, Inc.).

Statistical analysis. Comparisons among multiple groups were analyzed using one-way ANOVA followed by Tukey's post-hoc test. Statistical analyses were performed using SPSS software (version 16.0; SPSS Inc.). $\mathrm{P}<0.05$ was considered to indicate a statistically significant difference. All experiments were repeated $\geq 3$ times. Data are presented as the mean \pm SD.

\section{Results}

Shikonin reduces TGF- $\beta$-induced fibrosis in LX-2 cells. Cell viability was evaluated using the CCK- 8 assay. Cells were treated with $0.1-0.4 \mu \mathrm{mol} / 1$ shikonin for $24 \mathrm{~h}$. The results indicated that 0.1 and $0.2 \mu \mathrm{mol} / 1$ shikonin did not significantly alter LX-2 cell viability compared with the control group (Fig. 1A). By contrast, 0.3 and $0.4 \mu \mathrm{mol} / 1$ shikonin significantly reduced LX-2 cell viability compared with the control group (Fig. 1A). Additionally, the results of the EdU incorporation assay indicated that $0.2 \mu \mathrm{mol} / 1$ shikonin had no significant effect on LX-2 cell viability compared with the control group (Fig. 1B). The cytotoxicity of shikonin is a major index of its safety, which is a prerequisite for clinical application; therefore, a non-toxic concentration $(0.2 \mu \mathrm{mol} / \mathrm{l})$ of shikonin was used for subsequent experiments. To observe the effect of shikonin on 

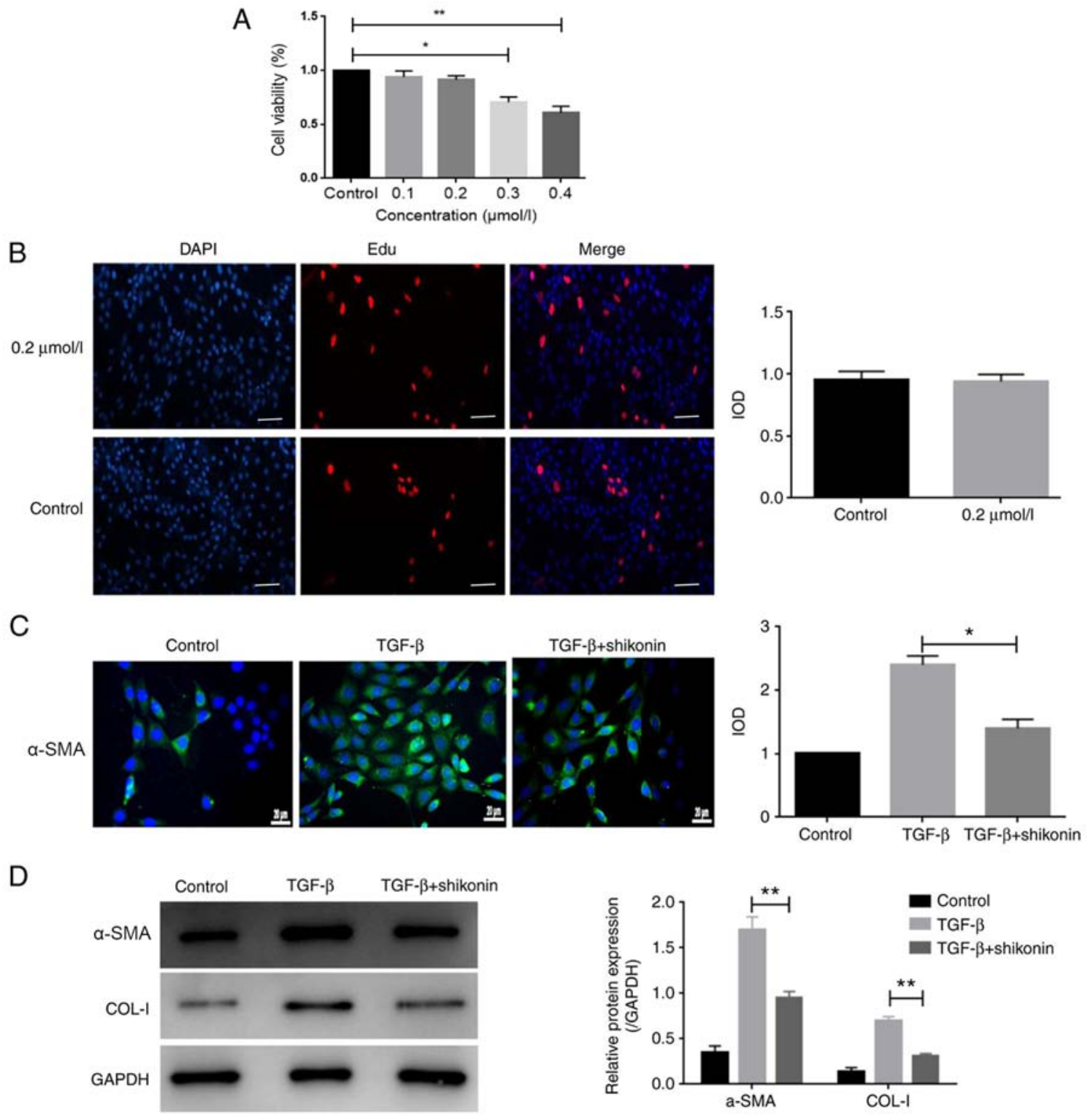

Figure 1. Shikonin inhibits TGF- $\beta$-induced fibrosis in LX-2 cells. (A) Cell viability was assessed using the Cell Counting Kit-8 assay. (B) Representative images of the EdU incorporation assay (magnification, x100). (C) The expression of $\alpha$-SMA was assessed via immunofluorescence. (D) The expression of $\alpha$-SMA and COL-I was detected via western blotting. ${ }^{*} \mathrm{P}<0.05$ and ${ }^{* * *} \mathrm{P}<0.01$ vs. control. $\alpha$-SMA, $\alpha$ smooth muscle actin; COL-I, type I collagen; IOD, integrated optical density.

fibrosis in LX-2 cells, the expression of $\alpha$-SMA was measured. The protein expression levels of $\alpha$-SMA and COL-I were markedly increased by TGF- $\beta$ and shikonin significantly decreased TGF- $\beta$-induced protein expression (Fig. $1 \mathrm{C}$ and D). The results indicated that shikonin markedly inhibited fibrosis.

Shikonin promotes apoptosis in TGF- $\beta$-treated LX-2 cells. The effect of shikonin on cell apoptosis was examined. LX-2 cells were treated with shikonin for $24 \mathrm{~h}$ and the effect of shikonin on LX-2 cell apoptosis was determined by flow cytometry. Compared with the control group, TGF- $\beta$ treatment decreased LX-2 cell apoptosis, whereas shikonin treatment significantly decreased TGF- $\beta$-induced LX-2 cell apoptosis (Fig. 2A). Additionally, western blotting was performed to detect the expression of apoptosis-related proteins, including caspase-3, Bcl- 2 and Bax. Compared with the control group, TGF- $\beta$ increased the expression levels of caspase- 3 and $\mathrm{Bcl}-2$, and decreased the expression levels of Bax. In contrast, shikonin significantly decreased TGF- $\beta$-induced alterations in protein expression (Fig. 2B).

Shikonin inhibits autophagy in TGF- $\beta$-stimulated LX-2 cells. Autophagy serves an important role in fibrosis (10). Therefore, whether shikonin exerted a regulatory effect on autophagy was investigated. The expression of autophagy-related proteins (LC3-I, LC3-II, Beclin-1 and P62) was determined. The expression levels of LC3-II/I and Beclin-1 were significantly increased, whereas P62 expression levels were significantly decreased in the TGF- $\beta$ group compared with the control group (Fig. 3A). Moreover, shikonin significantly inhibited autophagy, as indicated by reduced expression levels of LC3-II/I and Beclin-1, and increased P62 expression level in the TGF- $\beta+$ shikonin group compared with the TGF- $\beta$ group. Compared with the TGF- $\beta$ group, the number of 

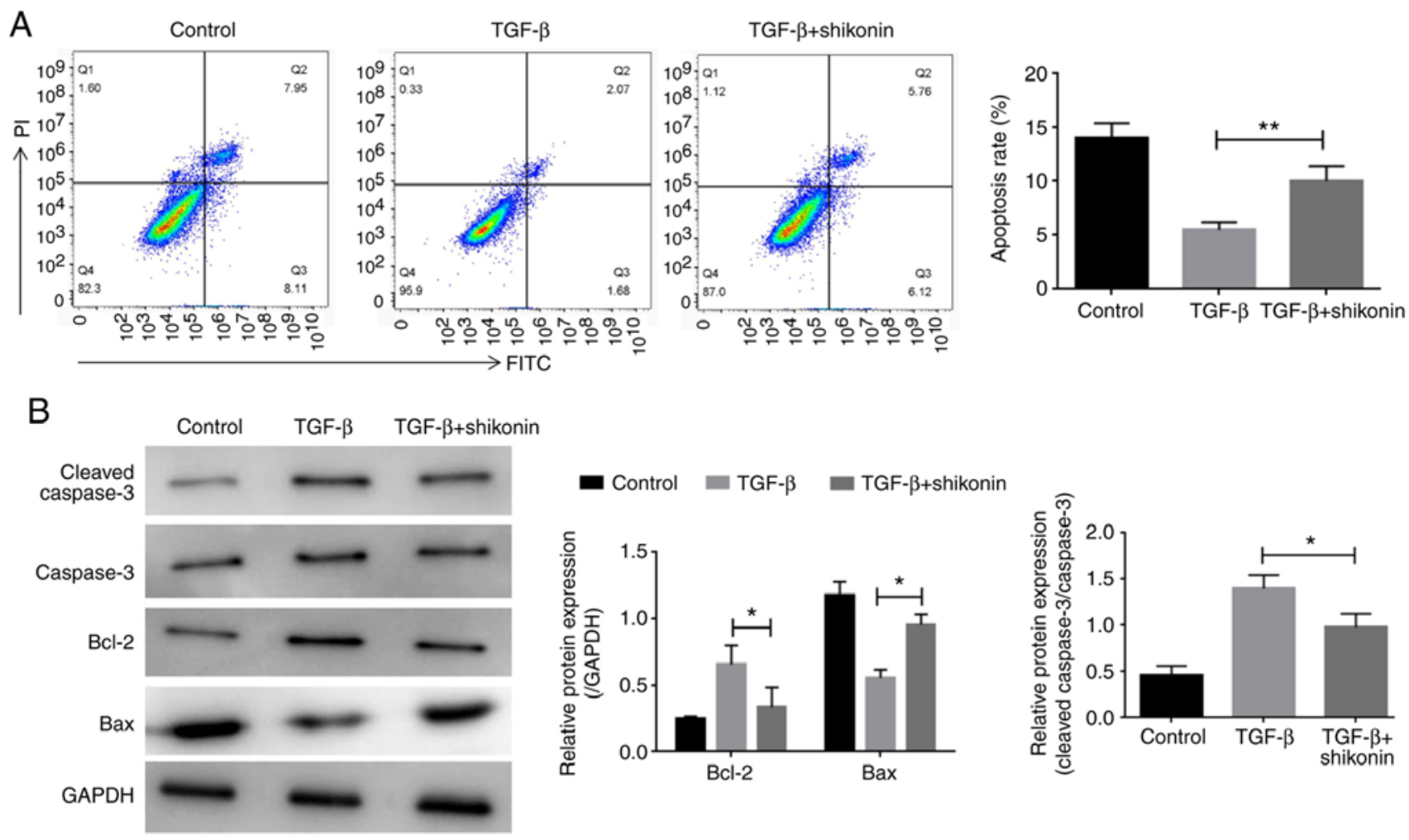

Figure 2. Effect of shikonin on cell apoptosis. (A) Cell apoptosis was assessed via flow cytometry. (B) The protein expression levels of caspase-3, Bcl-2 and Bax were measured via western blotting. ${ }^{*} \mathrm{P}<0.05$ and ${ }^{* *} \mathrm{P}<0.01$.
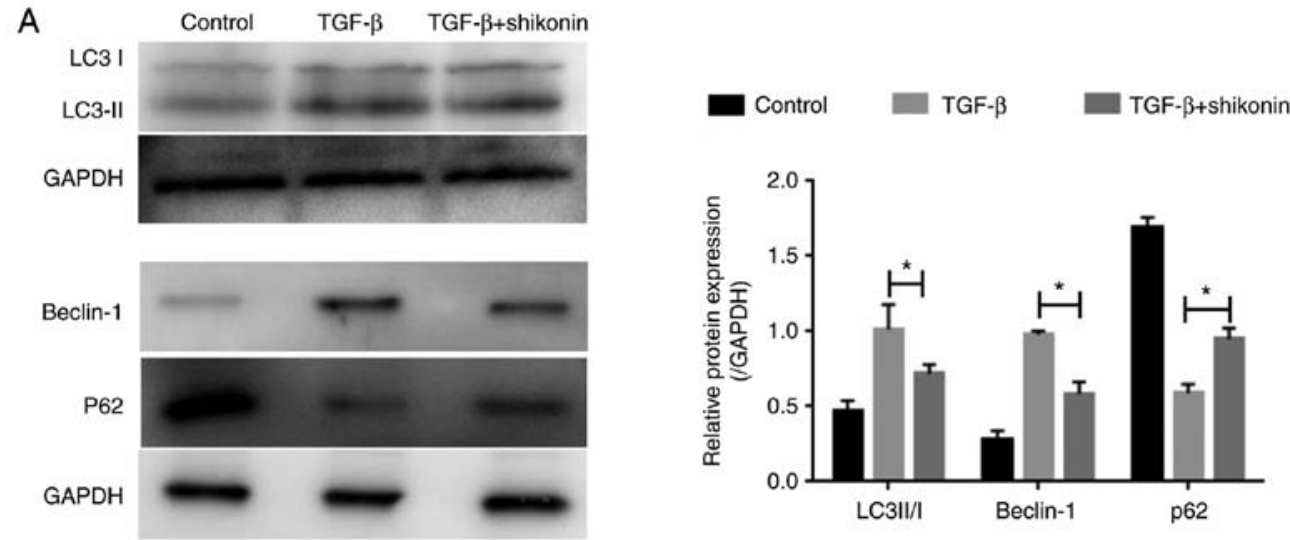

B
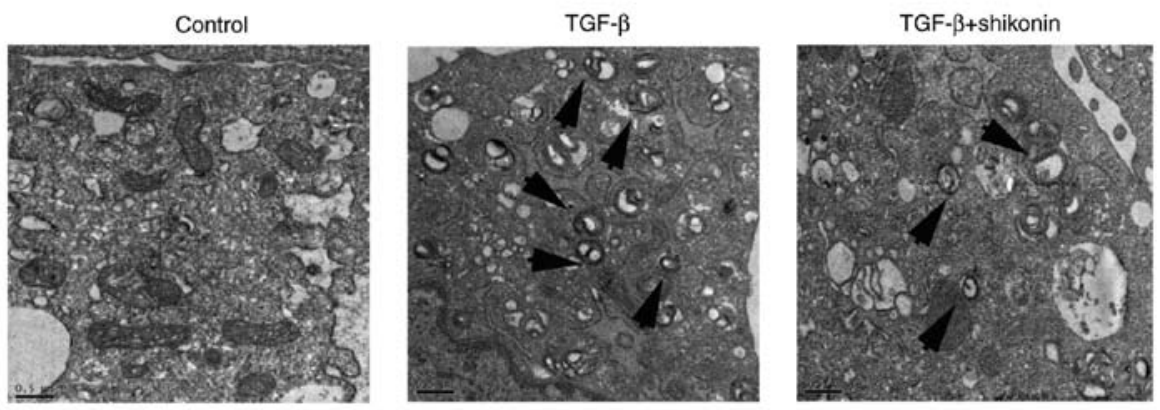

Figure 3. Effect of shikonin on LX-2 cell autophagy. (A) The protein expression levels of LC3-II/I, Beclin-1 and P62 were measured via western blotting. (B) Representative images of autophagosomes (indicated by arrows) obtained via transmission electron microscopy. "P<0.05. LC 3, light chain 3.

autophagosomes in the shikonin group was notably reduced (Fig. 3B). The results indicated that the inhibitory effect of shikonin on fibrosis in LX-2 cells may be mediated via suppressing autophagy. 
A
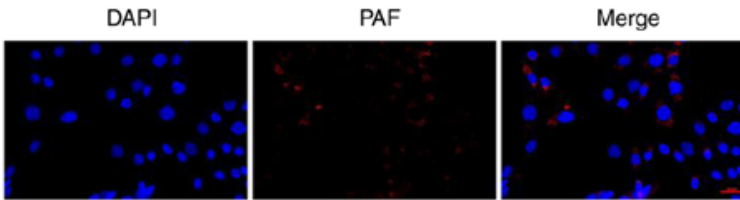

TGF- $\beta$
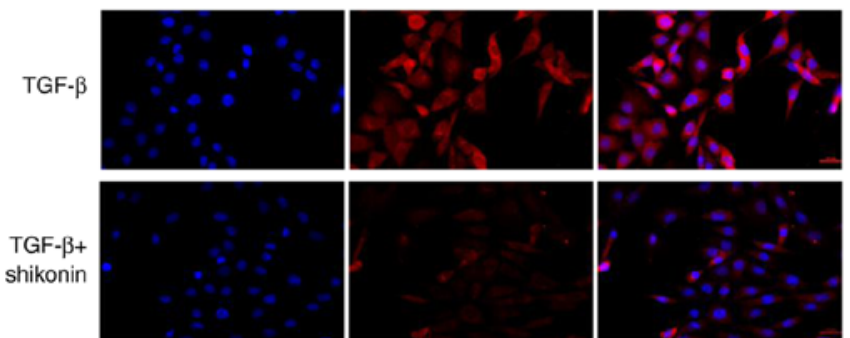

B
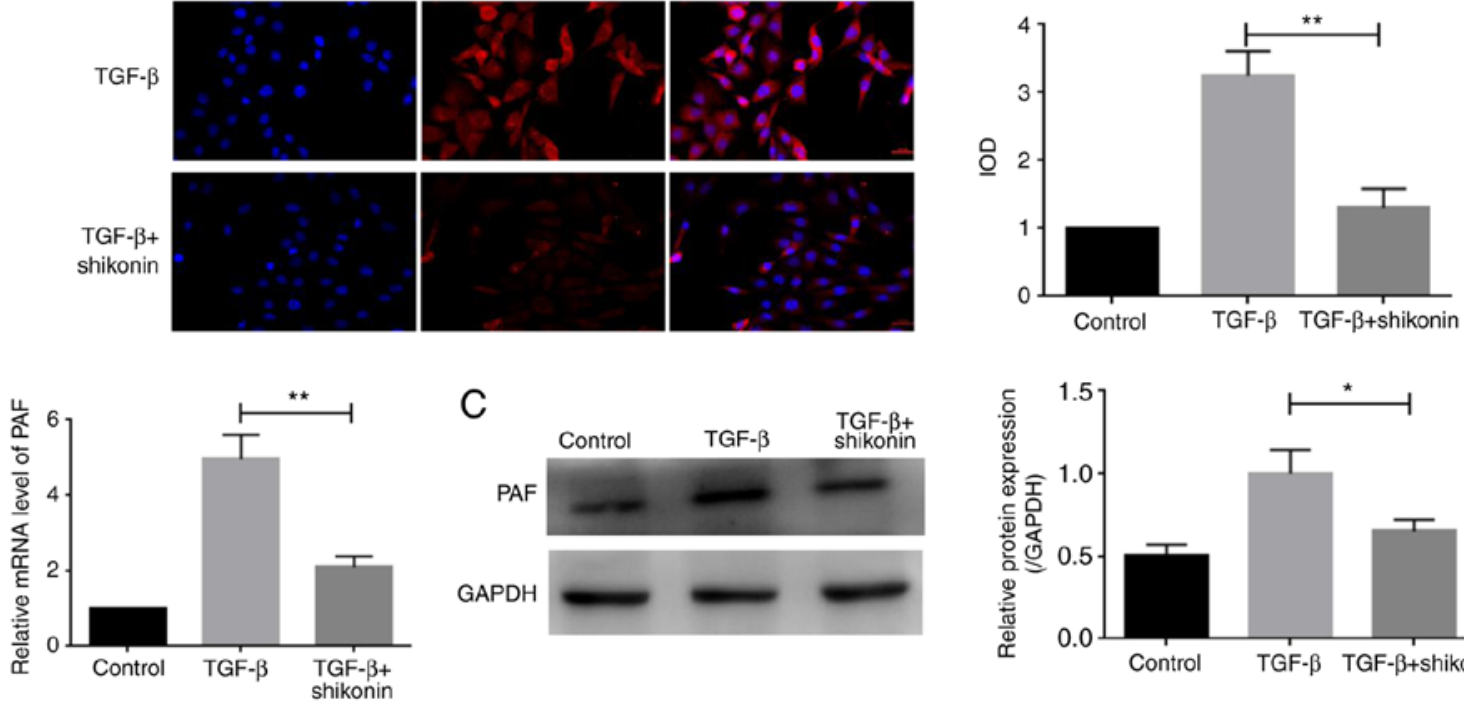

Figure 4. Effect of shikonin on the expression of PAF. (A) The expression of PAF was measured via immunofluorescence (magnification, $\mathrm{x} 200$ ). (B) The mRNA expression levels of PAF. (C) The protein expression level of PAF was assessed via western blotting. $\mathrm{P}<0.05$ and ${ }^{* *} \mathrm{P}<0.01$. PAF, platelet-activating factor; IOD, integrated optical density.
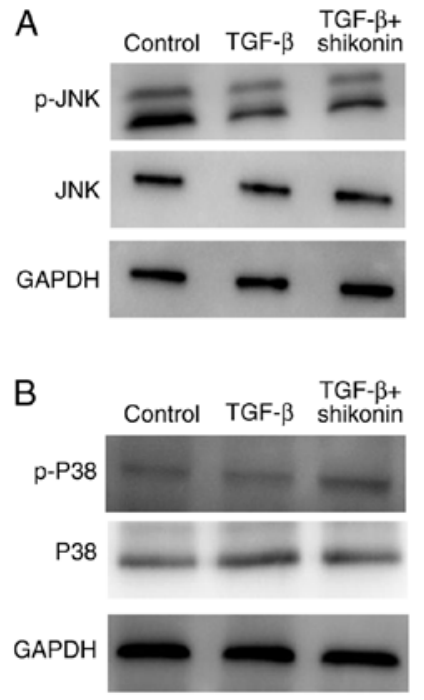
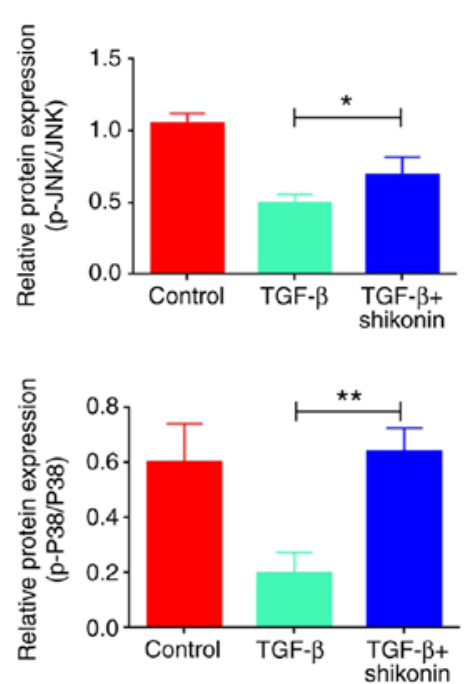

C

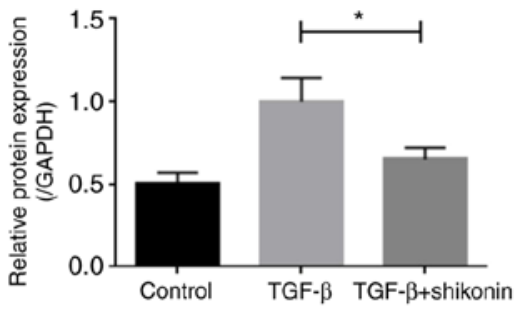

Figure 5. Effects of shikonin on the expression levels of p-JNK, JNK, p-P38 and P38. The expression levels of (A) p-JNK, JNK, (B) p-P38 and P38 were assessed via western blotting. (C) Graphic illustrating the collective results of the present study. ${ }^{*} \mathrm{P}<0.05$ and ${ }^{* *} \mathrm{P}<0.01$. p-, phosphorylated; HSC, hepatic stellate cell; ECM, extracellular matrix.

Shikonin reduces the expression of PAF in $L X-2$ cells. PAF serves a key role in cell apoptosis and autophagy (5). The expression of PAF was determined in the present study. PAF levels were higher in the TGF- $\beta$ group compared with the control group. PAF expression was significantly decreased in the shikonin + TGF- $\beta$ group compared with the TGF- $\beta$ group (Fig. 4A). Additionally, the western blotting and RT-qPCR results indicated an inhibitory effect of shikonin on PAF expression (Fig. 4B and C).
Shikonin activates MAPK signaling by upregulating the expression levels of $p-J N K$ and $p$-P38. The mechanisms of action underlying the effects of shikonin on cell apoptosis and autophagy were evaluated. A previous study reported that PAF regulated cell apoptosis via the MAPK signaling pathway (5). Therefore, whether shikonin affected MAPK signaling was investigated. The results indicated that TGF- $\beta$ inhibited the MAPK signaling pathway, as indicated by downregulated p-JNK and p-P38 expression levels in the TGF- $\beta$ group 
compared with the control group, which were significantly upregulated by shikonin treatment (Fig. 5).

\section{Discussion}

Liver fibrosis is a compensatory reaction in the process of tissue repair following liver injury and inflammation caused by various chronic pathogenic factors (1). During chronic liver injury, increased ECM secretion and decreased degradation can lead to excessive deposition of ECM, which eventually results in liver fibrosis (1). Activated HSCs are the major producers of ECM (1). In the present study, TGF- $\beta$ was used to induce LX-2 cell activation to establish a hepatic fibrosis model. Shikonin induced apoptosis and inhibited autophagy, thereby inhibiting liver fibrosis development. Moreover, shikonin inhibited PAF expression and may serve a role in inhibiting liver fibrosis via the MAPK signaling pathway.

Shikonin, a compound extracted from natural lithospermum, has been reported to have a wide range of biological and pharmacological properties. Previous studies have demonstrated that shikonin exerts antibacterial, antitumor, anti-inflammatory and antifibrosis effects (11-16). Certain studies (16-19) demonstrated that shikonin improved isoproterenol-induced myocardial injury by inhibiting myocardial fibrosis, inflammation, apoptosis and endoplasmic reticulum stress. Wang et al (13) revealed that shikonin inhibited the occurrence and development of pancreatic cancer via the NF- $\kappa \mathrm{B}$ signaling pathway. Jing et al (20) reported that shikonin induced HaCaT cell apoptosis via the mitochondrial, Erk and Akt signaling pathways. In the present study, the results indicated that shikonin may inhibit the development of liver fibrosis by inducing HSC apoptosis, indicating that shikonin may serve as a therapeutic agent for liver fibrosis.

Autophagy is an important strategy to maintain cell homeostasis (21). Autophagy is active in liver cells and maintains the normal physiological function of liver cells by eliminating easily accumulated proteins and damaged mitochondria (21-23). Additionally, autophagy is an important regulator implicated in various liver diseases (22). Although autophagy mediates the adaptive mechanism against injury via degradation of the damaged organelles and proteins produced in liver diseases, it may also serve as a promoter triggering the development and progression of liver disease (22). Moreover, it has been demonstrated that autophagosomes in HSCs promote the decomposition of lipid droplets into free fatty acids in patients with liver fibrosis, indicating that autophagy is involved in the physiological process of liver fibrosis (22). Shen et al (16) reported that astaxanthin improved liver fibrosis by regulating TGF-1 expression and the process of autophagy. In the present study, shikonin significantly reduced the protein expression levels of LC3-II/I and Beclin-1, and the TEM results indicated that the number of autophagosomes was significantly reduced, suggesting that shikonin may delay the process of liver fibrosis by inhibiting autophagy.

PAF is a bioactive lipid neurotransmitter that acts on various cells and tissues and serves an important role in the pathogenesis of several diseases (24). Liang et al (24) reported that IncRNA pyruvate formate-lyase regulated the expression of PAF by serving as a competitive endogenous let-7d RNA, thereby regulating the progression of myocardial fibrosis. Lv et al (25) demonstrated that rupatadine inhibited pulmonary fibrosis by acting on PAF. The present study revealed that shikonin significantly reduced the expression of PAF via the MAPK signaling pathway in a cell model of liver fibrosis, indicating that shikonin may inhibit liver fibrosis by regulating the expression of PAF.

In summary, the results reported that shikonin induced HSC apoptosis and inhibited autophagy by acting on the PAF protein, thus blocking the development of liver fibrosis. Therefore, the present study indicated a potential novel therapeutic strategy for liver fibrosis. However, animal experiments are required to verify the effect of shikonin in liver fibrosis.

\section{Acknowledgements}

Not applicable.

\section{Funding}

The present study was supported by the Wuhan Health Bureau Research Project (grant no. WX09C07).

\section{Availability of data and materials}

The datasets used and/or analyzed during the present study are available from the corresponding author on reasonable request.

\section{Authors' contributions}

MS and $\mathrm{HZ}$ designed and performed the experiments, analyzed data and wrote the manuscript. ZC and JY performed the experiments. JLi and SS performed parts of the experiments. JLiu participated in the experimental design, provided financial support and revised the manuscript. All authors read and approved the final manuscript.

\section{Ethics approval and consent to participate}

Not applicable.

\section{Patient consent for publication}

Not applicable.

\section{Competing interests}

The authors declare that they have no competing interests.

\section{References}

1. Friedman SL: Evolving challenges in hepatic fibrosis. Nat Rev Gastroenterol Hepatol 7: 425-436, 2010.

2. Zhang X, Cui JH, Meng QQ, Li SS, Zhou W and Xiao S: Advance in anti-tumor mechanisms of shikonin, alkannin and their derivatives. Mini Rev Med Chem 18: 164-172, 2018.

3. Hernandez-Gea V and Friedman SL: Pathogenesis of liver fibrosis. Annu Rev Pathol 6: 425-456, 2011.

4. Ma X, Yu M, Hao C and Yang W: Shikonin induces tumor apoptosis in glioma cells via endoplasmic reticulum stress, and Bax/Bak mediated mitochondrial outer membrane permeability. J Ethnopharmacol 263: 113059, 2020. 
5. Guo C, He J, Song X, Tan L, Wang M, Jiang P, Li Y, Cao Z and Peng C: Pharmacological properties and derivatives of shikonin-A review in recent years. Pharmacol Res 149: 104463 , 2019.

6. Nie Y, Yang Y, Zhang J, Cai G, Chang Y, Chai G and Guo C: Shikonin suppresses pulmonary fibroblasts proliferation and activation by regulating Akt and p38 MAPK signaling pathways. Biomed Pharmacother 95: 1119-1128, 2017.

7. Ding H, Jiang L, Xu J, Bai F, Zhou Y, Yuan Q, Luo J, Zen K and Yang J: Inhibiting aerobic glycolysis suppresses renal interstitial fibroblast activation and renal fibrosis. Am J Physiol Renal Physiol 313: F561-F575, 2017

8. Fan C, Dong Y, Xie Y, Su Y, Zhang X, Leavesley D and Upton Z: Shikonin reduces TGF- $\beta 1$-induced collagen production and contraction in hypertrophic scar-derived human skin fibroblasts. Int J Mol Med 36: 985-991, 2015.

9. Yang J, Wang Z and Chen DL: Shikonin ameliorates isoproterenol (ISO)-induced myocardial damage through suppressing fibrosis, inflammation, apoptosis and ER stress. Biomed Pharmacother 93: 1343-1357, 2017.

10. Wang $\mathrm{H}$ and Zhang G: Activation of CaMKK $\beta$-AMPK-mTOR pathway is required for autophagy induction by $\beta, \beta$-dimethylacrylshikonin against lung adenocarcinoma cells. Biochem Biophys Res Commun 517: 477-483, 2019.

11. Tian R, Li Y and Gao M: Shikonin causes cell cycle arrest and induces apoptosis by regulating the EGFR/NF- $\kappa B$ signaling pathway in human epidermoid carcinoma A431 cells. Biosci Rep 35: e00189, 2015.

12. Wei Y, Li M, Cui S, Wang D, Zhang CY, Zen K and Li L: Shikonin inhibits the proliferation of human breast cancer cells by reducing tumor-derived exosomes. Molecules 21: 777, 2016.

13. Wang Y, Zhou Y, Jia G, Han B, Liu J, Teng Y, Lv J, Song Z, Li Y, Ji L, et al: Shikonin suppresses tumor growth and synergizes with gemcitabine in a pancreatic cancer xenograft model: Involvement of NF- $\kappa$ B signaling pathway. Biochem Pharmacol 88: 322-333, 2014.

14. Wang L, Gai P, Xu R, Zheng Y, Lv S, Li Y and Liu S: Shikonin protects chondrocytes from interleukin-lbeta-induced apoptosis by regulating PI3K/Akt signaling pathway. Int J Clin Exp Pathol 8: 298-308, 2015.

15. Wada N, Kawano Y, Fujiwara S, Kikukawa Y, Okuno Y, Tasaki M, Ueda M, Ando Y, Yoshinaga K, Ri M, et al: Shikonin, dually functions as a proteasome inhibitor and a necroptosis inducer in multiple myeloma cells. Int J Oncol 46: 963-972, 2015
16. Shen M, Chen K, Lu J, Cheng P, Xu L, Dai W, Wang F, He L, Zhang Y, Chengfen $\mathrm{W}$, et al: Protective effect of astaxanthin on liver fibrosis through modulation of TGF- $\beta 1$ expression and autophagy. Mediators Inflamm 2014: 954502, 2014.

17. Zhu XF, Un XF, Liu GH and Chen FF: Effects of shikonin on the proliferation, migration and differentiation of $\mathrm{HaCaT}$ cells mediated by IL-22. J Clin Dermatol 43: 215-218, 2014.

18. Qisen Li, Zeng J, Su M, He Y and Zhu B: Acetylshikonin from Zicao attenuates cognitive impairment and hippocampus senescence in D-galactose-induced aging mouse model via upregulating the expression of SIRT1. Brain Res Bulletin 137: 311-318, 2018.

19. Wang $H$ and Zhang G: Endoplasmic reticulum stress-mediated autophagy protects against $\beta, \beta$-dimethylacrylshikonin-ind uced apoptosis in lung adenocarcinoma cells. Cancer Sci 109: 1889-1901, 2018

20. Jing H, Sun W, Fan J, Zhang Y, Yang J, Jia J, Li J, Guo J, Luo S and Zheng Y: Shikonin induces apoptosis of HaCaT cells via the mitochondrial, Erk and Akt pathways. Mol Med Rep 13: 3009-3016, 2016

21. He C and Klionsky DJ: Regulation mechanisms and signaling pathways of autophagy. Annual Rev Genet 43: 67-93, 2009.

22. Mallat A, Lodder J, Teixeira-Clerc F, Moreau R, Codogno P and Lotersztajn S: Autophagy: A multifaceted partner in liver fibrosis. Biomed Res Int 2014: 869390, 2014. https://doi. org/10.1155/2014/869390.

23. Zhang XW, Zhou JC, Peng D, Hua F, Li K, Yu JJ, Lv XX, Cui B, Liu SS, Yu JM, et al: Disrupting the TRIB3-SQSTM1 interaction reduces liver fibrosis by restoring autophagy and suppressing exosome-mediated HSC activation. Autophagy 16: 782-796, 2020.

24. Liang H, Pan Z, Zhao X, Liu L, Sun J, Su X, Xu C, Zhou Y, Zhao D, Xu B, et al: LncRNA PFL contributes to cardiac fibrosis by acting as a competing endogenous RNA of let-7d. Theranostics 8: 1180-1194, 2018.

25. Lv XX, Wang XX, Li K, Wang ZY, Li Z, Lv Q, Fu XM and $\mathrm{Hu}$ ZW: Rupatadine protects against pulmonary fibrosis by attenuating PAF-mediated senescence in rodents. PLoS One 8: e68631, 2013 